\title{
Resolución de problemas y comprensión situacional
}

\author{
SANTIAGO VICENTE Y JOSETXU ORRANTIA* \\ Universidad de Salamanca

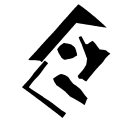 \\ Resumen
}

Las matemáticas y la resolución de problemas son herramientas básicas en la escolaridad de las sociedades avanzadas, y en su aprendizaje intervienen una serie de procesos cognitivos. En este articulo se presenta una revisión de los modelos teóricos y de los estudios empíricos que dan cuenta de cómo la aplicación de conocimientos sobre el mundo real, adquiridos durante la trayectoria vital de los sujetos, interviene en la resolución de problemas. Para ello, en primer lugar se describen los diferentes tipos de problemas de matemáticas; en segundo lugar se revisan los modelos cognitivos que justifican la necesidad de aplicar conocimientos sobre el mundo real para generar un modelo de la situación para resolverlos; en tercer lugar se revisan e interpretan los resultados de los estudios que han comprobado la validez de esos modelos teóricos, dando al mismo tiempo claves para la mejora del rendimiento de los alumnos. Finalmente se exponen las conclusiones generales de la revisión y se aportan algunas implicaciones para la práctica educativa que se desprenden de esas conclusiones

Palabras clave: Resolución de problemas matemáticos, modelos de la situación, reescritura de problemas, modelos cognitivos.

\section{Word problem solving and situational knowledge}

\begin{abstract}
Mathematics and word problem solving are basic tools employed in the schooling of advanced societies and their learning involves various cognitive processes. The present paper reviews theoretical models and empirical studies that explain how the application of knowledge about the real world, acquired during subjects' lives, mediates in problem solving. For this purpose, first, different types of mathematical word problems are described; second, cognitive theoretical models that justify the need of applying knowledge about the real world in order to generate a situation model for children to successfully solve word problems are described; third, empirical studies that have shown the validity of these theoretical models are analysed and their results discussed with a view to improving student performance. Finally, general conclusions obtained from the review are put forth and some educational implications based on these conclusions are presented.
\end{abstract}

Keywords: Word problem solving, situation models, rewording, cognitive models.

Agradecimientos: Este trabajo ha sido posible gracias al Proyecto SEJ2006-11249/EDUC, financiado por el Ministerio de Educación y Ciencia. Asimismo, agradecemos sinceramente a Montserrat Castelló y a Manuel Montanero su generosidad y sus comentarios sobre la primera versión del manuscrito.

Correspondencia con los autores: Santiago Vicente. Universidad de Salamanca. Facultad de Educación. Pso. Canalejas 169.37008 Salamanca.E-mail: sanvicente@usal.es Original recibido: Enero, 2007; Aceptado: Febrero, 2007. 
Resolver problemas de matemáticas es una de las herramientas fundamentales de las sociedades avanzadas, en cuyo aprendizaje la escuela invierte buena parte de su tiempo y esfuerzo. Tal es así que podríamos considerarla, junto a la lectura, una de las capacidades que la escuela ha tratado de universalizar. Sin embargo, con frecuencia se hacen públicos indicadores que señalan que los alumnos muestran un nivel en matemáticas en general y en resolución de problemas en particular preocupantemente bajo (p.e.: Informe PISA, ver INECSE, 2004).

¿Cuáles pueden ser los motivos de este preocupantemente bajo nivel de rendimiento? Uno de los motivos podría ser que a pesar de que esta tarea pudiera parecer relativamente sencilla desde el punto de vista cognitivo, los procesos cognitivos implicados en el proceso de resolución de problemas matemáticos son muchos y muy complejos. Otro motivo, estrechamente relacionado con el anterior, podría ser que, a pesar de esta complejidad, la cultura de aula frecuentemente presenta los problemas de matemáticas de una manera estereotipada, limitando el proceso de resolución a sus aspectos estrictamente matemáticos, de manera que potencia el desarrollo de los procesos vinculados a los conocimientos estrictamente matemáticos a la vez que relega otros, relacionados con la aplicación de otros tipos de conocimiento y que en ocasiones son igualmente importantes. En esta revisión señalamos la necesidad de alejar la práctica educativa de este enfoque de la enseñanza de la resolución de problemas, basándonos en una serie de modelos teóricos y de estudios empíricos que apuntan a la necesidad de presentar los problemas de matemáticas como una tarea de aplicación de conocimientos no sólo matemáticos, sino también sobre el mundo real y de sentido común, que son necesarios para una comprensión genuina de la situación descrita por el problema.

El objetivo de este artículo es doble. Por un lado, revisaremos los modelos cognitivos de resolución de problemas que incluyen esta comprensión situacional como parte del proceso de resolución, y los estudios empíricos que han comprobado la validez de esos modelos. En segundo lugar, propondremos una serie de conclusiones y posibles orientaciones para la práctica educativa.

Para ello hemos estructurado este trabajo en cuatro partes diferentes. En la primera de ellas delimitamos los diferentes tipos de problemas a los que los alumnos pueden enfrentarse, y cuál es la influencia de la aplicación del conocimiento situacional en cada uno de ellos. Una vez delimitada esta cuestión, expondremos brevemente, en segundo lugar, qué modelos teóricos han sido propuestos para justificar la implicación de la comprensión situacional en el proceso de resolución. En tercer lugar, describiremos los estudios empíricos que ha comprobado la validez de estos modelos teóricos, aportando al mismo tiempo claves para potenciar esta comprensión situacional. Finalmente, proponemos una serie de conclusiones, a la luz de la interpretación de los resultados obtenidos, sobre la implicación de la comprensión situacional en la resolución de problemas y, por lo tanto, de la validez de los modelos teóricos, así como una serie de implicaciones educativas.

\section{Tipos de problemas}

Para aprender a resolver problemas, a lo largo de su trayectoria escolar un alumno habrá de enfrentarse a diferentes tareas matemáticas. Para resolver algunas de ellas necesitará conocimientos exclusivamente matemáticos, mientras que para resolver otras, además de esos conocimientos matemáticos, necesitará otros acerca del mundo real, adquiridos a través de su experiencia vital. De esta manera, los problemas matemáticos que los estudiantes deben resolver en las tareas 
escolares pueden clasificarse en un continuo, en función de la relevancia que adquiere para resolverlos la comprensión situacional.

\section{Problemas verbales realistas}

Los problemas de aplicación realista o auténticos problemas son aquellos que reproducen fielmente situaciones del mundo real. Se trata de problemas que necesitan de un razonamiento basado en el conocimiento sobre el mundo real. De ahí que la utilización de procedimientos aritméticos pueda, incluso, conducir a soluciones que, siendo correctas desde el punto de vista matemático, carezcan realmente de sentido. Verschaffel, De Corte y Lassure (1994) proponen una clasificación en cinco categorías que hemos reorganizado en la tabla I.

TABLA I

Tipos de problemas realistas, adaptado de Verschaffel et al. (1994)

\begin{tabular}{l|l}
\hline \multicolumn{1}{c|}{ Razonamiento } & \multicolumn{1}{c}{ Ejemplos } \\
\hline $\begin{array}{l}\text { Juntar o separar conjuntos } \\
\text { que pueden tener } \\
\text { elementos comunes }\end{array}$ & $\begin{array}{l}\text { Juan tiene } 5 \text { amigos y Pedro tiene } 6 \text { amigos. Juan y Pedro } \\
\text { deciden hacer una fiesta juntos. Ellos invitan a todos sus } \\
\text { amigos. Todos los amigos están presentes. ¿Cuántos amigos } \\
\text { hay en la fiesta? }\end{array}$ \\
\cline { 2 - 2 } &
\end{tabular}

Roberto y Alicia van a la misma escuela. Roberto vive a 17 kilómetros de la escuela y Alicia a $8 \mathrm{~km}$. ¿A qué distancia vive Roberto de Alicia?

Considerar elementos relevantes que no aparecen explícitamente en el problema

Roberto ha comprado 4 tablones de 2,5 m. cada uno. Cuántos tablones de $1 \mathrm{~m}$ pueden sacar de estos tablones?

Un hombre quiere tener una cuerda los suficientemente larga para unir dos postes separados entre si 12 metros, pero solo tiene trozos de cuerda de 1,5 metros. ¿Cuántos trozos necesitaría juntar para hacer la cuerda lo suficientemente larga para unir las estacas?

Sumar o restar 1 al Si la escuela de Villaseco se inauguró el 1 de enero de 1964 y resultado estamos en el año 2007, ¿cuántos años lleva abierta la escuela?

Interpretar el resto de una división no exacta

450 soldados deben ser transportados a su lugar de entrenamiento. En cada autobús pueden entrar 36 soldados ¿Cuántos autobuses serán necesarios?

El abuelo da a sus 4 nietos una caja con 18 globos para repartir entre ellos. ¿Cuántos globos le toca a cada uno?

Decidir una solución de proporcionalidad directa Juan corre los 100 metros en 17 segundos. ¿Cuánto tardará en correr 1 kilómetro? o no

Este recipiente se está llenando con un grifo a un ritmo constante. Si el agua tiene una profundidad de $4 \mathrm{~cm}$ tras 10 segundos, ¿cuánta profundidad tendrá después de 30 segundos? (este problema se acompaña por un recipiente de forma cónica)

\section{Problemas verbales contextualizados que requieren conocimientos matemáticos}

Los "story word problems" son problemas verbales en los que se contextualizan en una situación problemática que ha de resolverse a través de operaciones 
algebraicas o aritméticas. A diferencia de los problemas realistas, es posible (al menos en determinadas ocasiones) resolverlos sin apelar a conocimientos no matemáticos. Sin embargo, determinados modelos teóricos sostienen que también es necesario comprender la situación descrita por el problema para resolverlo correctamente, y generar un modelo de la situación del problema, como veremos más adelante.

Los problemas algebraicos proponen situaciones relativamente complejas, cuya resolución implica el manejo de expresiones compuestas de constantes y variables (número y letras). Siguiendo a Mayer (1981), pueden clasificarse en ocho familias, de las cuales las más relevantes, y que comprenden la mayoría de estos problemas, son los problemas de razón (cantidad por tiempo, coste por unidad, parte del coste total y cantidad de cantidad), de geometría (como los problemas de áreas) y de estadística. La tabla II propone un ejemplo de cada uno de ellos.

TABLA II

Ejemplos de los principales tipos de problemas de álgebra propuestos por Mayer (1981)

\begin{tabular}{l|l}
\hline Tipo de problema & \multicolumn{1}{c}{ Ejemplos } \\
\hline De razón & $\begin{array}{l}\text { Una hormiga gigante está aterrorizando a la ciudad de San } \\
\text { Francisco. Viaja hacia el este en dirección a Detroit, que está } \\
\text { a dos mil cuatrocientas millas de distancia, a una velocidad } \\
\text { de cuatrocientas millas por hora. El ejército se percata de esto } \\
\text { una hora después y envía un helicóptero al oeste desde Detroit } \\
\text { a seiscientas millas por hora para interceptar a la hormiga. } \\
\text { Si la hormiga salió a las 2 p.m. a qué hora colisionarán la } \\
\text { hormiga y el helicóptero? (Tomado de Nathan, Kintsch y } \\
\text { Young, 1992, p. 349) }\end{array}$ \\
\hline De geometría & $\begin{array}{l}\text { Un señor compró un solar cuadrado en el centro del pueblo de } \\
36 \text { metros de lado para hacerse una vivienda. Pagó 112.750 } \\
\text { euros el metro cuadrado. ¿Cuánto dinero ha invertido en el } \\
\text { solar? }\end{array}$ \\
\hline De estadística & $\begin{array}{l}\text { Un juego consiste en tirar dos dados. Si la suma de sus caras es } \\
\text { mayor o igual a 10 se ganan 30 céntimos, si está comprendida } \\
\text { entre 7 y 9 seganan 10 céntimos. y para cualquier otro } \\
\text { resultado no se gana nada. ¿Cuál debería ser el precio de la } \\
\text { apuesta para que la ganancia esperada de la banca sea de } \\
50 \text { céntimos? }\end{array}$ \\
\hline
\end{tabular}

Los problemas aritméticos, por su parte, son más sencillos que los algebraicos y se resuelven mediante la aplicación de operaciones aritméticas sencillas. Estos problemas pueden clasificarse en función de su estructura matemática. Siguiendo a Heller y Greeno (1978), podemos establecer una distinción entre problemas de cambio (aquellos en los que una cantidad inicial sufre un cambio y da lugar a una cantidad final), de comparación (en los que una cantidad referente se compara con otra, dando lugar a un conjunto diferencia entre ambos) y de combinación (en los que dos cantidades o partes se combinan para dar lugar a una tercera, o todo). Algunos autores añaden una cuarta categoría, de igualación, que supone una mezcla de las estructuras de comparación y cambio, ya que se formulan en términos de que cantidad hay que añadir a un conjunto (referente) para igualarlo a otro conjunto (comparado). En la siguiente tabla se ofrecen ejemplos de cada uno de estos subtipos. 
TABLA III

Ejemplos de los tres tipos de problemas propuestos por Heller y Greeno (1978)

\begin{tabular}{l|l}
\hline Tipo de problema & \multicolumn{1}{c}{ Ejemplos } \\
\hline De cambio & $\begin{array}{l}\text { Pedro quería renovar la instalación eléctrica de su casa. } \\
\text { Pedro tenía algunos metros de cable que le habían sobrado } \\
\text { de una instalación anterior. Como Pedro se dio cuenta de que } \\
\text { esos metros de cable no serían suficientes para toda la } \\
\text { instalación compró 75 metros de cable más. Después de } \\
\text { comprar el cable tenía 117 metros. Entonces Pedro se } \\
\text { preguntó ¿Cuántos metros de cable tenía al principio? } \\
\text { (Adaptado de Vicente, Orrantia y Verschaffel, en prensa) }\end{array}$ \\
\hline De comparación & $\begin{array}{l}\text { Juan y Pedro han ido a una fiesta de cumpleaños. Juan tiene } \\
8 \text { caramelos y Pedro } 5 \text { menos. ¿Cuántas tiene Juanito? }\end{array}$ \\
\hline De combinación & $\begin{array}{l}\text { Luis y Andrés tienen 9 caramelos entre los dos, 3 de ellos } \\
\text { son de Luis. ¿Cuántos tiene Andrés? }\end{array}$ \\
\hline
\end{tabular}

\section{Resolución de operaciones aritméticas}

En el polo opuesto a los problemas realistas podríamos situar aquellos ejercicios que no se asocian a ningún contexto situacional concreto, sino que únicamente requieren la resolución de operaciones aritméticas, del tipo " $9+8$ ". Diversos trabajos han propuesto modelos evolutivos de resolución de operaciones aritméticas básicas, en las que únicamente influye la adquisición de conocimiento matemático (para una revisión, ver Baroody, 1993, 1999; Baroody y Gannon, 1984; Baroody, Wilkins y Tiilikainen, 2003; Canobi, Reeve y Pattison, 2002; Fuson, 1988, 1992; Fuson, Secada y Hall, 1983; Resnick, 1983, 1989, 1992; Sophian, Harley y Martin, 1995).

Una vez delimitadas las principales tareas matemáticas a las que se enfrentarán los alumnos a lo largo de la Educación Primaria, describiremos en las siguientes páginas los modelos teóricos que se han propuesto desde la Psicología cognitiva para explicar los procesos mentales implicados en su resolución.

\section{Modelos teóricos}

La resolución de problemas ha atraído la atención tanto de educadores como de psicólogos cognitivos desde hace muchos años. Desde el ámbito de la Psicología Cognitiva se han propuesto una serie de modelos teóricos para explicar los procesos cognitivos implicados en la resolución de problemas, y que pueden categorizarse en dos grandes grupos, en función del tipo de proceso cognitivo en el que se han centrado. El primero de ellos ha aportado información sobre cómo los alumnos comprenden la estructura matemática de los problemas, y cómo esta comprensión matemática les permite determinar qué operación aritmética es necesaria para resolverlo (para una revisión de estos modelos, ver Orrantia, 2003). Por otro lado, un segundo conjunto de estudios se ha interesado en comprobar si la comprensión de la situación en la que se inserta el problema, mediante la activación y la aplicación de conocimientos previos sobre el mundo real, influye en el proceso de resolución y, si es así, en qué grado lo hace. Sin embargo, mientras que necesidad de comprender la estructura matemática de los problemas ha sido ampliamente documentada, los modelos y estudios dedicados a documentar la necesidad de entender la situación denotada por el proble- 
ma ha recibido menor atención. Es precisamente en este segundo tipo en el que nos centraremos en esta revisión.

La necesidad de crear un modelo de la situación del problema, aplicando para ello el conocimiento del mundo real que posea el alumno, ha sido justificada desde diferentes modelos teóricos para cada uno de los tipos de problemas. Todos ellos, tanto los propuestos para los problemas realistas como para los problemas algebraicos y aritméticos, se sustentan en la idea de que antes de generar una representación mental de la estructura matemática del problema es necesario representar previamente la situación propuesta por el mismo.

De esta manera, en primer lugar, para los problemas realistas, Verschaffel, Greer y De Corte (2000) proponen un modelo según el cual, para resolver el problema, el sujeto, primero, ha de comprender la situación descrita por el problema; después, debe construir un modelo matemático que recoja los elementos esenciales de esa situación problemática y de las relaciones existentes entre ellos, para luego extraer las implicaciones que se derivan de ese modelo matemático para interpretar los resultados obtenidos, y por último evaluar esta interpretación respecto al modelo de la situación previamente generado y comunicar el resultado del proceso de resolución.

Por ejemplo, tomando el problema de los autobuses (Tabla I), el sujeto, en primer lugar ha de comprender que todos los soldados han de ser transportados, que debe haber suficientes autobuses, que cada autobús tiene un límite de plazas, que en cada autobús no puede ir más de ese número máximo de personas... En segundo lugar, de ese modelo de la situación debe deducir que si el total de soldados son 450 y que en cada autobús caben 36, el número de autobuses necesario vendrá dado por la división de 450 entre 36, dando como resultado 12.5. Después el alumno ha de interpretar este resultado en función del modelo de la situación, y percatarse de que el número de autobuses ha de ser un número entero, y de que es necesario transportar a todos los soldados, de manera que la solución correcta no es 12.5 sino 13 autobuses. Una vez realizado este razonamiento, el alumno será ya capaz de dar una respuesta al problema.

Esta necesidad de generar un modelo de la situación cualitativa del problema antes de extraer su esencia matemática subyace también a los modelos teóricos relativos a la resolución de los problemas algebraicos y aritméticos. Para la resolución de los primeros, Nathan Kintsch y Young (1992) proponen un modelo teórico según el cual para resolver el problema, en primer lugar, el sujeto ha de leer y comprender el enunciado. Una vez comprendido, debe generar tanto una representación cualitativa del problema (modelo de la situación) mediante el uso de sus conocimientos previos sobre el mundo real, como un modelo algebraico del problema (modelo matemático) utilizando conocimientos previos de tipo algebraico. Retomando el problema de la hormiga, el sujeto, en primer lugar, debe crear una representación mental de los personajes, en este caso, la hormiga y el helicóptero, situándolos en la dirección adecuada y recreando una distancia entre sí de acuerdo con la descripción de la situación propuesta por el problema. Una vez creada esta representación, el sujeto debe elegir la estructura matemática que de adecue a la situación planteada (en este caso, cantidad X tiempo) y situar los datos del problema dentro de esa estructura para, en un último momento, ejecutar las operaciones necesarias para resolverlo.

En el caso de los problemas aritméticos, dos modelos plantearon la necesidad de crear una representación mental de la situación del problema. El primero de ellos fue el Situation Problem Solver (en adelante S.P.S.) de Reusser (1988), un modelo computacional (esto es, un programa informático capaz de resolver los problemas a partir de una serie de parámetros) basado en una propuesta anterior desarrollada por Kintsch y Greeno (1985). Mientras que el modelo de Kintsch y 
Greeno resolvía los problemas generando dos niveles representacionales diferentes - un texto base proposicional y un modelo de la situación estrechamente relacionado con la estructura matemática del problema-, el de Reusser es capaz de generar verdaderas representaciones cualitativas de la situación, superando así la limitación principal del modelo de Kintsch y Greeno (1985) y acercándose a la esencia de los propuestos por Verschaffel et al. (2000) y Nathan et al. (1992). Reusser propone que entre el texto base y el modelo matemático del problema es necesario generar el Modelo Episódico de la Situación (en adelante M.E.S.), de carácter estrictamente cualitativo. Según este modelo, el proceso de resolución puede dividirse en cinco pasos, que se ilustran en la figura 1.

En un primer momento, el modelo comprende el texto, a través de la construcción del texto base, esto es, de la representación proposicional del texto del problema, en los mismos términos que el modelo de Kintsch y Greeno (1985). En un segundo momento, el modelo genera el M.E.S, una representación cualitativa de la estructura temporal y funcional de las acciones y situaciones que se describen en el problema, y en el que también se incluye la representación de los agentes, las acciones y las relaciones entre los eventos que evoca el problema. Para generar esta representación, de manera similar a cualquier otro tipo de texto, el modelo utiliza la información contenida en el texto base, y en el caso de que sea necesario, determinadas inferencias a partir de sus conocimientos previos en relación con el campo de conocimiento de referencia (van Dijk y Kintsch, 1983). Reusser considera que la generación de este modelo cualitativo de la situación es imprescindible para el proceso de resolución, y sostiene que hasta que el modelo no ha generado el M.E.S. la comprensión no es lo suficientemente profunda como para ejecutar los pasos de abstracción que dan lugar a la matematización del problema y a la posterior reducción del M.E.S. al modelo matemático del problema, esto es, a la representación mental de su estructura matemática.

En tercer lugar, el modelo construye una representación de la estructura matemática del problema (el "modelo del problema"), aplicando su conocimiento matemático. Una vez activado el conocimiento matemático necesario, el modelo proyecta el M.E.S. sobre éste, de manera que las relaciones situacionales representadas en el M.E.S. se transformen en relaciones matemáticas que permitan dar respuesta a la pregunta formulada por el problema.

En el cuarto momento del proceso el modelo reduce el modelo del problema a su esencia matemática abstracta, en forma de ecuación matemática. Por último, el modelo genera una respuesta volviendo al modelo de la situación y dando un significado semántico a la respuesta del problema.

Volviendo al ejemplo de problema aritmético de cambio planteado en la tabla III, el S.P.S. comenzaría resolviendo el problema mediante la transformación del texto del problema en un conjunto de proposiciones. Tras ello, el modelo crearía el M.E.S., en el que se representaría la secuencia temporal de la situación (al principio tenía algo de cable, luego compró más cable, y al final tiene otra cantidad de cable), la estructura causal (necesitaba cable porque quería cambiar la instalación, compró más porque no tenía suficiente) y las intenciones de los personajes (quería cambiar la instalación eléctrica de su casa). Una vez creado este nivel representacional el modelo extrae su esencia matemática, y proyecta esta información cualitativa en un modelo matemático del problema consistente en un esquema de cambio con tres huecos, uno para cada conjunto. Tras ello, abstrae la ecuación del problema $(\mathrm{X}+75$ = 117) y aplica el conocimiento matemático para ejecutar la operación (en este caso, restar)

De este modo, la clave del modelo SPS es que el proceso de matematización del problema ya no se basa directamente en el texto base del problema, sino que 
FIGURA 1

Proceso de resolución de problemas según el modelo S.P.S. de Reusser (1988). Adaptado de Staub y Reusser (1995)

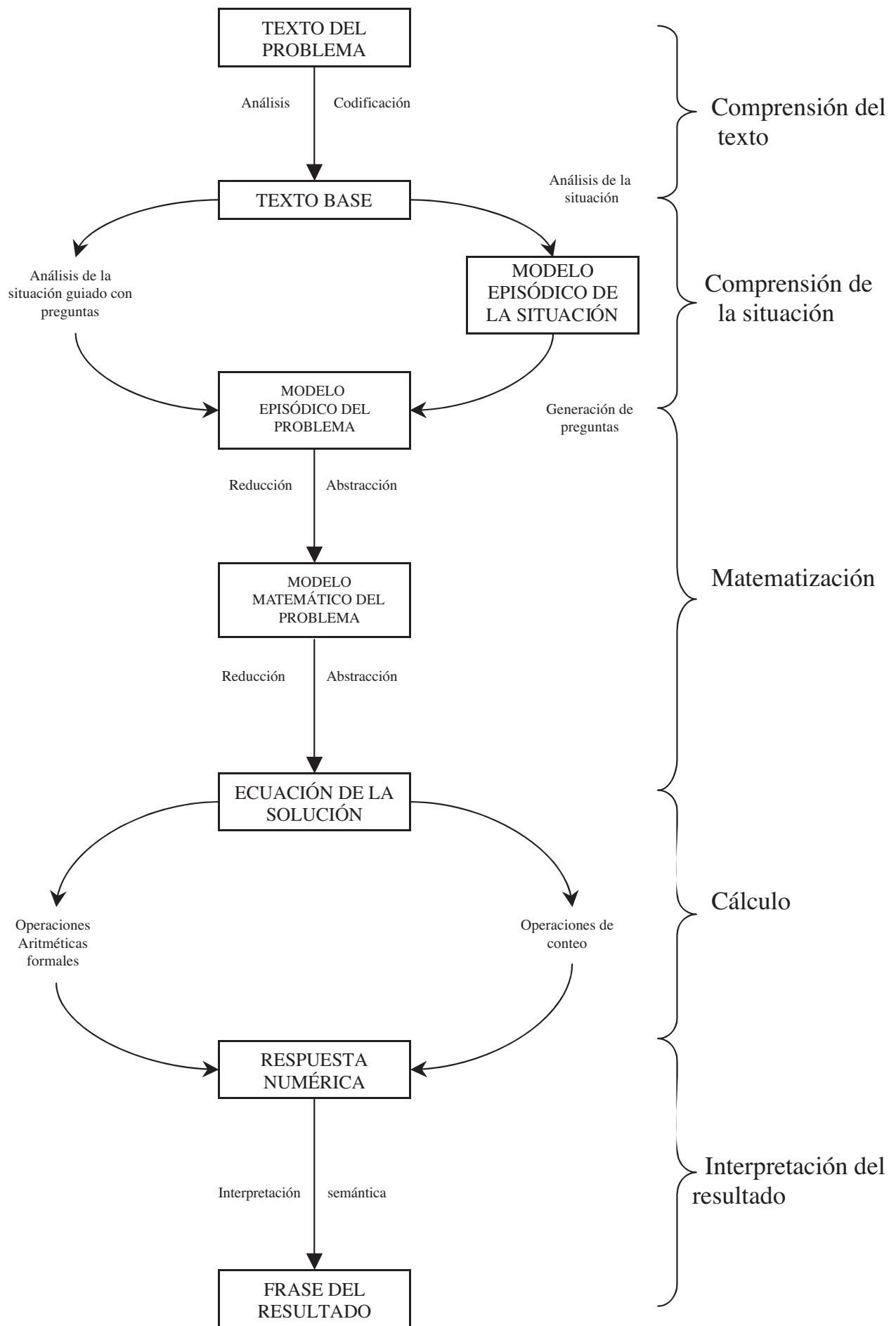


se extrae del M.E.S. del que, en un momento posterior, se deduce la estructura matemática del problema.

El segundo modelo computacional que considera la necesidad de aplicar el conocimiento sobre el mundo real en la resolución de problemas es el desarrollado por Kintsch (1988). Este modelo complementó el modelo de Reusser (1988) al proponer una explicación alternativa a la influencia del conocimiento sobre el mundo real. Al igual que el de Kintsch y Greeno (1985), este modelo resuelve los problemas generando dos niveles representacionales, texto base y modelo del problema, pero de manera radicalmente diferente al modelo de Kintsch y Greeno (1985). En un primer momento, de Construcción, tanto en el texto base como en el modelo de la situación no se generan únicamente las proposiciones presentes en el texto del problema, sino también las que, procedentes del conocimiento previo sobre el mundo real que posea el sujeto ${ }^{1}$, están relacionadas con el enunciado del problema (siguiendo el ejemplo de problema propuesto, estas proposiciones podrían ser del tipo "la instalación está anticuada", "Pedro es electricista” etcétera). En un segundo momento, de Integración, se eliminan las proposiciones con bajo nivel de activación, esto es, las que menos relacionadas están con el resto, y se genera finalmente una representación en la que sólo se preservan las proposiciones altamente relacionadas entre sí, independientemente de si formaban parte del enunciado del problema o si fueron inferidas desde el conocimiento previo. Así, esos conocimientos sobre el mundo del sujeto contribuyen a que el alumno represente (y, por lo tanto, comprenda) los problemas de matemáticas.

En síntesis, desde el ámbito de la Psicología Cognitiva se han propuesto modelos que justifican la importancia de la comprensión cualitativa de la situación en la que el problema se halla inserto para el proceso de resolución, tanto de los problemas realistas (Verschaffel et al., 2000), como para los problemas de álgebra (Nathan et al., 1992), como para los problemas aritméticos (Kintsch, 1988, 1998; Reusser, 1988). En esencia, todos estos modelos proponen la necesidad de generar un modelo cualitativo de la situación del problema, esto es una representación de la situación descrita por el problema, para lo cual es necesario activar conocimientos previos acerca del mundo real y del sentido común.

\section{Estudios empíricos}

Los modelos teóricos que acabamos de describir son claros al señalar la necesidad de crear un modelo cualitativo de la situación para resolver problemas no solo realistas, sino también algebraicos y aritméticos. Con el objetivo de comprobar la importancia de la activación de conocimientos sobre el mundo real para la creación de modelos de la situación en el rendimiento de los alumnos se han desarrollado una serie de estudios empíricos que, en unas ocasiones, han corroborado las conclusiones derivadas de esos modelos mientras que en otros casos han ofrecido resultados menos acordes con ellos.

\section{Descripción de los estudios empíricos}

Comenzando por los problemas realistas, multitud de estudios han documentado el fenómeno de la "suspensión del sentido" en la resolución de problemas realistas (Caldwell, 1995; Carpenter, Lindquist, Matthews y Silver, 1983; Greer, 1993; Hidalgo, 1997; Radatz, 1983; Renkl, 1999; Reusser y Stebler, 1997; Säljö y Wyndhamn, 1990; Semandeni, 1995; Silver, Shapiro y Deutch, 1993; Verschaffel, De Corte y Lassure, 1999; Verschaffel et al., 1994; Yoshida, Verschaffel y De Corte, 1997). En todos estos estudios la gran mayoría de los alumnos resolvieron este tipo de problemas simplemente ejecutando las operaciones 
matemáticas necesarias, sin realizar ningún tipo de consideración sobre la adecuación de la respuesta obtenida a la situación real propuesta. De esta manera, aún habiendo generado un buen modelo matemático del problema, los alumnos omitían deliberadamente las implicaciones derivadas de la aplicación de su conocimiento sobre el mundo real al dar una respuesta al problema, lo cual impedía la creación de un modelo cualitativo adecuado y les llevaba a aportar respuestas que carecían de sentido desde el punto de vista situacional.

En cuanto a los problemas de álgebra, dos estudios instruccionales (Jonassen, 2003; Nathan et al., 1992) avalan la eficacia de promover la generación de un modelo mental cualitativo en la resolución de problemas de álgebra. Nathan $e t$ al. (1992) diseñaron una aplicación informática tutorial, denominada "Animate”, basada en el modelo teórico que señalamos unas líneas más arriba. Esta aplicación, implementada para la resolución de problemas de álgebra de razón, en primer lugar pedía al alumno que generase una representación gráfica explícita del modelo de la situación del problema, seleccionando personajes de entre un conjunto de pequeños dibujos que los representaban, y orientando cada uno de ellos en una dirección determinada, de acuerdo con la situación propuesta por el problema. Tras ello, pedía al alumno que seleccionase una representación esquemática de la estructura matemática (en este caso, algebraica) del problema. Después, proporcionaba un vínculo entre el modelo de la situación y el modelo del problema por medio de una animación que, integrando el modelo de la situación y el modelo matemático del problema, ilustraba las acciones de los personajes indicadas por el sujeto en su representación del problema. La figura 2 representa este proceso

Por otra parte, Jonassen (2003) desarrolló una aplicación informática similar a la de Nathan et al. (1992), que compartía la misma idea y cuyo funcionamiento era muy similar, si bien en este caso el modelo de la situación que el sujeto generaba al manipular los datos se mostraba al sujeto a través de imágenes estáticas, en lugar de animaciones. Tanto los resultados de la implementación del programa de Nathan et al. (1992) como los del de Jonassen (2003), indicaron la alta efectividad que este tipo de programas tiene sobre el acierto con el que los sujetos resuelven los problemas a los que se enfrentan.

Los estudios empíricos que acabamos de describir apuntan a que tanto los problemas realistas como los problemas de álgebra requieren del sujeto cierto esfuerzo de interpretación más allá de lo matemático para poder ser resueltos con éxito. ¿Qué ocurre con los problemas aritméticos? Determinados modelos teóricos clásicos (Briars y Larkin, 1984; Riley, Greeno y Heller, 1983; también Kintsch y Greeno, 1985), sostienen que es posible que estos problemas puedan resolverse únicamente utilizando conocimientos matemáticos. No obstante, como ya hemos señalado, los modelos más recientes de resolución de problemas aritméticos (Kintsch, 1988; Reusser, 1988) discrepan de estos modelos clásicos y sostienen que la generación de un modelo de la situación con información no sólo matemática, sino del mundo real, es un paso necesario para la resolución con éxito de los problemas.

¿Cuál es entonces la influencia de la creación de un modelo de la situación en la resolución de problemas aritméticos? Para responder a esta cuestión se han desarrollado una serie de estudios empíricos que, a través de la reescritura de los enunciados de los problemas, tratan de comprobar si incluyendo información de tipo situacional en el texto del problema los alumnos son capaces de generar un modelo de la situación más completo y, con ello, resolverlo con más eficacia.

El trabajo pionero en el campo de la reescritura de problemas es el desarrollado por Hudson (1983). Este autor sostiene que los problemas de comparación son más difíciles que los de cambio o combinación porque la com- 
FIGURA 2

Representación esquemática del modelo ANIMATE, adaptado de Nathan et al. (1992)

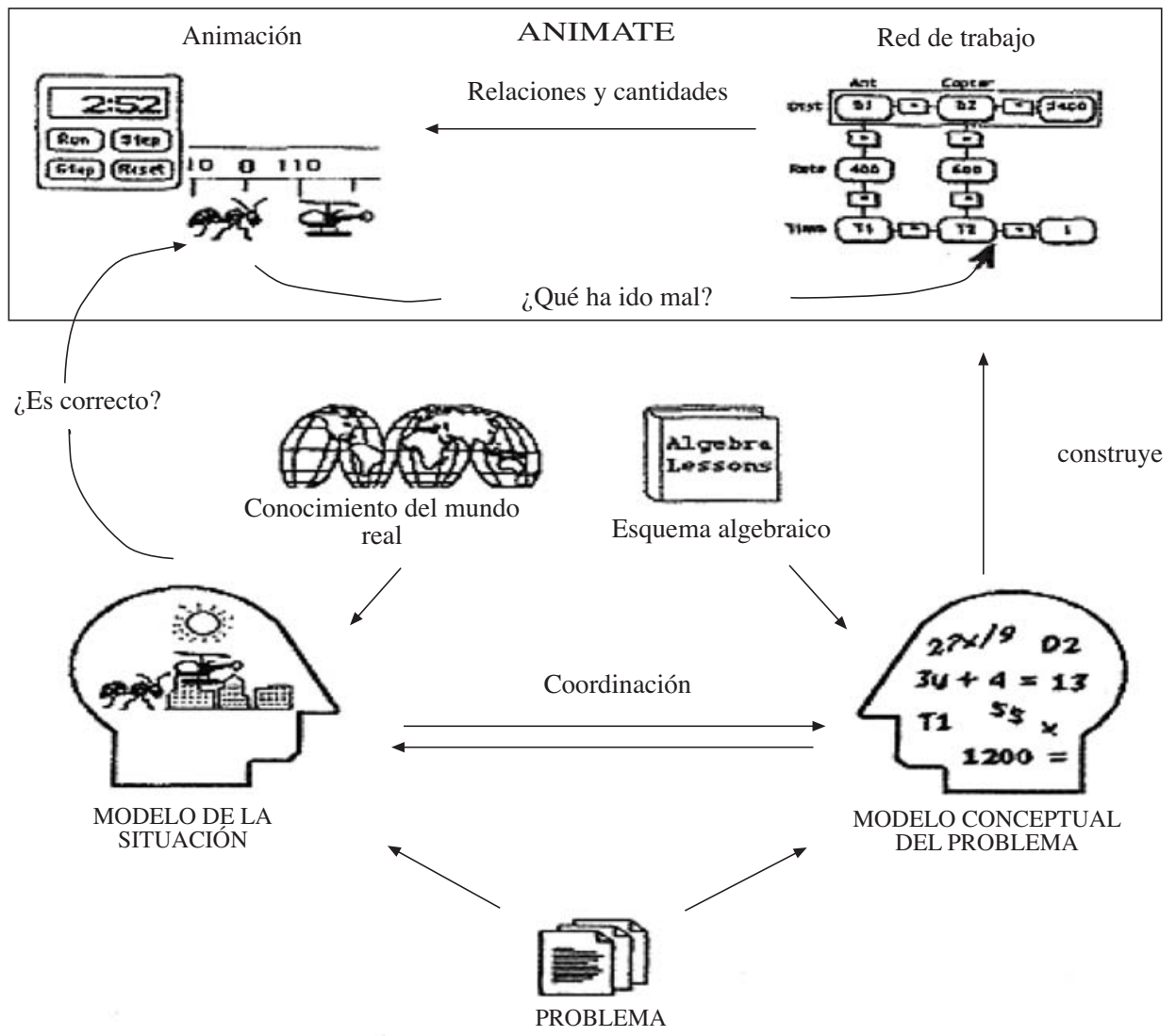

prensión del termino comparativo $<$ mas que $>$ es problemática (Hudson, 1983 , p. 84). Para sortear esta dificultad el autor propuso una tarea que ha sido frecuentemente replicada (p.e.: De Corte, Verschaffel y De Win, 1985; Fan, Mueller y Marini, 1994), y que se basa en la sustitución de la pregunta típica de los problemas de comparación con la diferencia desconocida ("Aquí hay algunos pájaros y algunos gusanos. ¿Cuantos pájaros más que gusanos hay?") por otra en la que se evita ese término comparativo ("Aquí hay algunos pájaros y algunos gusanos. Supón que los pájaros compiten entre ellos y cada uno intenta coger un gusano. ¿Tendrán todos los pájaros un gusano? ¿Cuántos pájaros no tendrán un gusano?”). Los resultados de la aplicación de esta tarea en alumnos de Educación Infantil y de primero de Primaria mostraron que los niños del estudio resolvían con mucha más facilidad el problema reescrito que su versión "estándar". Hudson atribuyó los resultados a que, en los problemas "estándar" los niños interpretaron de manera errónea la expresión “¿Cuantos más que...?”. Sin embargo, Staub y Reusser (1995) proponen una explicación alternativa: esta reescritura, además de modificar las características lingüísticas del enunciado del problema, cambiaba el contexto situacional que envuelve el problema, haciéndolo más dinámico y comprensible para los alumnos, al estar más relacionados con su experiencia vital. De esta manera, estos autores vieron en estos resultados positivos un 
claro ejemplo de cómo modificando el contexto situacional descrito por el problema los alumnos son capaces de resolverlos de manera más efectiva.

Un segundo estudio fue el realizado por Cummins, Kintsch, Reusser y Weimer (1988). En este estudio los autores toman como base lo que denominaban problemas "empobrecidos", similares a los que hemos denominado "estándar" en el estudio de Hudson (1983), para elaborar a partir de ellos pequeñas historias, "que muestran situaciones plausibles y realistas" (p. 427), de entre 18 y 31 proposiciones de extensión ${ }^{2}$, según su análisis proposicional. Un ejemplo de estos problemas reescritos es el siguiente:

Bill lleva muchas cosas en sus bolsillos. Además, tiende a perder cosas todo el tiempo. Hoy de camino a su casa se le han caído 3 conchas. Cuando vació sus bolsillos ha encontrado sólo 6 conchas. Bill estaba triste porque su padre le había dado esas conchas. ¿Cuántas conchas tenía al principio? (Adaptado de Cummins et al., 1988, p. 429)

Los resultados indicaron, en contra de lo que los autores habían hipotetizado, que los problemas reescritos no suscitaban niveles de acierto significativamente superiores a los de los problemas "empobrecidos" en los niños mas pequeños del estudio (de $2^{\circ}$ curso).

El tercero de los estudios que se enmarcan en este conjunto de trabajos que han efectuado reescrituras que facilitan la creación de un modelo de la situación del problema es el de Davis-Dorsey, Ross y Morrison (1991). Este estudio incluyó dos tipos de reescritura, una diseñada para mejorar la comprensión de la situación del problema y otra para mejorar la comprensión matemática de problemas de cambio, combinación, y comparación. El primer tipo de reescritura difiere de los desarrollados por Hudson (1983) y por Cummins et al. (1988), ya que en este estudio la reescritura situacional consistió en personalizar los problemas sustituyendo los nombres de los personajes implicados en el problema por los de los propios alumnos e introduciendo información relacionada con ellos, obtenida de un cuestionario sobre sus gustos personales, nombres de amigos, actividades favoritas, etcétera. Por ejemplo, los autores tomaban un problema de combinación como el siguiente: "Juan ha caminado 3/5 de kilómetro para ver una película. Después ha caminado a casa de Miguel. Juan ha caminado en total 4/5 de kilómetro. ¿Cuanto ha caminado Juan desde el cine hasta la casa de Miguel?”

Basándose en este problema, los autores tomaban la información procedente del cuestionario y la incluían en él, de manera que el problema quedaba reescrito de la siguiente manera: “"Mejor amigo» ha caminado 3/5 de kilómetro para ver "película favorita». Después ha caminado a casa de «otro amigo». «Mejor amigo» ha caminado 4/5 de kilómetro en total. ¿Cuanto camino «mejor amigo» desde el cine hasta la casa de «otro amigo»?" Davis-Dorsey et al., 1991, p. 63.

El segundo tipo de reescritura introducido por los autores, y que no nos detendremos a explicar aquí, resaltaba la estructura matemática del problema (para una descripción más completa, ver Vicente, Orrantia y Verschaffel, en prensa). Los resultados indicaron que la personalización de los problemas incrementó el rendimiento de los niños de $5^{\circ}$ tanto en combinación con la reescritura matemática como de manera independiente, y en los niños de $2^{\circ}$ sólo en combinación de la reescritura matemática. Los autores atribuyeron los resultados obtenidos en los problemas personalizados a que estos problemas generaron una mayor motivación intrínseca que llevó a los alumnos a codificar mejor la información y a procesarla de manera más fácil. En definitiva, la personalización de los problemas incrementó la significatividad de la tarea y aumentó la conectividad entre el texto del problema y los esquemas mentales del niño. 
Otro estudio, realizado por Stern y Lehrndorfer (1992), propuso una reescritura diferente, en este caso sólo para problemas de comparación. Estos autores buscaban "manipular el contexto situacional, pero no las formulaciones verbales de los problemas" (Stern y Lehrndorfer, 1992, p. 262), para lo cual reescribieron los enunciados de los problemas de manera que mantenían el texto del problema inalterado, y le añadían, precediendo al problema, un contexto situacional, que podía ser de dos tipos. En primer lugar, este contexto podía ser una situación de competitividad entre los dos personajes:

Pedro es el hermano mayor de Laura. Como es mayor, su habitación es mayor y sus juguetes son más caros que los de Laura. Pedro además tiene una paga mayor que la de Laura y tiene una bici nueva mientras que la de Laura es la bici antigua de Pedro. Cuando Pedro hace sus deberes, Laura hace unos cuantos garabatos Pedro tiene 9 lápices, Laura tiene 4 lápices. ¿Cuántos lápices tiene Laura menos que Pedro? Stern y Lehrndorfer, 1992, p. 264

En segundo lugar, el contexto situacional podía no hacer alusión a ningún contexto de competitividad:

Berta y Lidia están en la misma clase en el colegio. Su profesora es la Sra. Álvarez. Ella hace muchas cosas agradables con los niños. Ayer fueron al zoo. Hoy están dibujando los animales. Berta tiene 6 lápices. Lidia tiene 4 lápices. ¿Cuantos lápices tiene Lidia menos que Berta? Stern y Lehrndorfer, 1992, p. 264

A su vez, en los problemas precedidos de contextos competitivos la relación entre este contexto competitivo y el problema podía ser de dos tipos: de compatibilidad, por ejemplo cuando en la pequeña historia precedente quien poseía el conjunto mayor era el personaje de más edad; o de incompatibilidad, cuando se planteaba la relación inversa, esto es, siguiendo el ejemplo anterior, cuando el dueño del conjunto mayor en el problema era el personaje de menor edad. Los resultados del estudio apuntaban que cuando los problemas iban precedidos por una historia competitiva resultaban más fáciles de resolver que si iban precedidos por un contexto neutro. Además, dentro de los contextos que proponían una situación de competitividad, fueron los problemas precedidos por contextos compatibles los que los alumnos resolvieron con más facilidad. A partir de estos resultados, los autores concluyeron que las dificultades que los niños experimentan con frecuencia al resolver problemas de comparación son debidas a problemas para reconocer el contexto en el que aparece la situación de comparación, lo cual a su vez hace que los niños no activen el conocimiento matemático necesario para resolver el problema. De este modo sitúa sus resultados en la línea de la teoría propuesta por Reusser (1988).

Por su parte, Staub y Reusser (1992) realizaron un estudio en el que introdujeron 4 modificaciones en el enunciado del problema que produjeron cambios importantes en el grado de acierto con el que los niños resolvían problemas aritméticos de cambio. A diferencia del estudio de Davis-Dorsey et al. (1991), en el que se crearon diferentes problemas para reescrituras diferentes, en el estudio de Staub y Reusser (1992) se introdujeron varias modificaciones en el mismo problema. Las modificaciones propuestas por los autores fueron cuatro. En primer lugar, para explicitar la existencia de un conjunto inicial en los problemas de cambio con el conjunto inicial desconocido, los autores introdujeron una acción a través de un verbo de transferencia-ganancia (en lugar de un estado estático de posesión), para que el niño activara su conocimiento general sobre los objetos y las posesiones. En segundo lugar, modificaron el orden en el que aparecían las acciones, de manera que en los problemas de este estudio la secuencia no coincidía con el orden natural de los acontecimientos. En tercer lugar el protagonista 
era el sujeto de todas las oraciones del enunciado del problema. Por último, se evito el uso de pronombres. La tabla II muestra un ejemplo de este conjunto de modificaciones propuestas por Reusser, comparados con los correspondientes problemas "estándar".

TABLA IV

Problemas reescritos en el estudio de Staub y Reusser (1992), p. 290

\begin{tabular}{ll}
\hline \multicolumn{1}{c}{ Cambio 1} & \multicolumn{1}{c}{ Cambio 6} \\
\hline Joe tenía 3 canicas & Joe tenía algunas canicas \\
Después Tom le dio 5 canicas más & Después le dio 5 canicas a Tom \\
¿Cuántas canicas tiene Tom ahora? & Ahora Tom tiene tres canicas \\
& ¿Cuántas canicas tenía Tom al principio? \\
& \\
Hoy Dane le dio 11 canicas a Susan & Peter tiene abora 4 manzanas \\
Ayer Dane se encontró 5 canicas & Ayer Peter le dio 7 manzanas a Mary \\
¿Cuántas canicas tiene Dane ahora? & ¿Cuántas manzanas tenía Peter ayer? \\
\hline
\end{tabular}

Los resultados indicaron que los problemas reescritos del estudio resultaron más difíciles que los problemas "estándar”. Además, los autores sugerían que el factor más influyente fue el segundo, esto es, la variación de la secuencia temporal.

En síntesis, de estos cinco estudios empíricos (salvo del de Cummins et al., 1988) podría deducirse que los resultados avalan la necesidad de crear un modelo de la situación del problema, empleando para ello los conocimientos previos sobre el mundo real, para resolver problemas aritméticos. Sin embargo, un análisis en profundidad de los resultados de estos trabajos nos permitirá comprobar que la mayoría de los estudios muestran limitaciones que comprometen las conclusiones a las que llegan sobre la necesidad de crear ese modelo de la situación para resolver los problemas. En las siguientes páginas describiremos estas limitaciones

\section{Re-análisis de los estudios empíricos}

Comenzando por el estudio de Hudson, si seguimos al autor la reescritura era útil porque permitía eludir el término comparativo “¿Cuántos....más que?”, y si nos guiamos por el reanálisis desarrollado por Staub y Reusser asumiremos que esta reescritura es eficaz porque propone un contexto situacional diferente, más comprensible para el alumno. Sin embargo, Vicente et al. (en prensa) proponen una tercera explicación: al cambiar la pregunta del problema por la pregunta “¿Cuántos... no tendrán?”, Hudson estaba introduciendo una acción dentro de una estructura general de comparación. Dicho de otro modo, estaba transformando la estructura matemática estática del problema de comparación a una estructura que recoge una acción dinámica dentro de una estructura estática, transformando así el problema de comparación en un problema de igualación, más fácil de resolver ya que indica una acción que el niño puede interpretar para comprender el problema y resolverlo correctamente (Fuson et al., 1997).

En segundo lugar, en el estudio de Stern y Lehndorfer (1992) es necesario considerar qué es lo que diferencia a los contextos comparativos de los contextos neutros, y los contextos comparativos compatibles de los incompatibles. Esta diferencia estriba en que la información adicional comparativa ayuda al niño a detectar la estructura matemática del problema, mientras que los contextos neutros proponen una situación de la que no puede deducirse estructura matemática 
alguna. Así, las diferencias entre los contextos comparativos y los contextos neutros estriba en que los primeros, como indicaban los autores, preparan al alumno para comprender la situación del problema, pero también su estructura matemática, mientras que los contextos neutros presentan al alumno una información que describe un contexto situacional "enriquecido" en términos de Cummins et al. (1988), pero irrelevante para la tarea.

En cuanto a las diferencias entre los contextos comparativos compatibles e incompatibles, el razonamiento es similar al anterior: en los contextos comparativos se le presenta información útil para determinar cuál es el conjunto mayor y cuál el menor, que es la pregunta que el alumno ha de responder para resolver el problema. Sin embargo, en los contextos incompatibles la información sobre qué conjunto es mayor y cuál es el menor no ayuda a responder a esa pregunta, de manera que este contexto ayuda al alumno a determinar la estructura matemática del problema pero no a determinar el rol matemático de los conjuntos, esto es, qué conjunto es el mayor y cuál es menor.

En definitiva, al igual que en la reescritura propuesta por Hudson, en el estudio de Stern y Lehndorfer los diferentes tipos de reescritura que surtieron efecto tienen mucho que ver con la estructura matemática del problema: si en el primero sosteníamos que la reescritura modificaba la estructura matemática, en el segundo hemos visto que la reescritura explicita esa estructura matemática. Además, los contextos compatibles señalan las relaciones matemáticas entre los conjuntos, de manera que podemos concluir que esta reescritura tiene que ver con el contexto situacional del problema, pero especialmente con el grado de explicitación de la estructura matemática del problema y de los roles de los conjuntos.

Prosiguiendo con nuestro re-análisis, en el estudio de Davis-Dorsey et al. (1991) la reescritura más eficaz no está relacionada tanto con la creación de un modelo cualitativo de la situación, como con la personalización el problema, lo cual, tal y como sostienen los autores, está relacionado en mayor medida con la motivación y, por añadidura, en el grado de activación del alumno y de la profundidad en el procesamiento (ver Mayer, Fennell, Farmer y Campbell, 2004) que con la creación de un modelo de la situación, entendido éste como la representación mental de la estructura temporal y causal del problema, siguiendo a Reusser (1988).

En cuanto al estudio de Staub y Reusser (1992), la reescritura mezcla modificaciones de diferentes tipos: sintácticas (rol del protagonista, uso de pronombres), matemáticas (explicitación del conjunto inicial) y situacionales (alteración del orden temporal de los acontecimientos). Además, como ya señalamos en su momento, se introducen todas las modificaciones a la vez, de manera que los resultados, tal y como señalan los autores, sólo pueden ser especulativos. Según los autores, la alteración del orden temporal influye claramente en el resultado, pero el diseño del estudio es tan confuso que es necesario tomar con cautela la interpretación de los resultados que aportan los autores.

Para finalizar este tercer apartado de nuestra revisión vamos a describir dos estudios recientes que han superado las limitaciones de los estudios previos. Estos estudios son los realizados por Moreau y Coquin-Viennot (2003) y por Vicente y colaboradores (Vicente, 2006; Vicente et al., en prensa).

El primero de estos trabajos, el desarrollado por Moreau y Coquin-Viennot (2003), trata de constatar la creación, durante el procesamiento de un problema aritmético, del modelo matemático del problema, por un lado, y del M.E.S. por otro, Para ello, las autoras reescribieron problemas de cambio de dos operaciones añadiendo sobre la estructura básica del problema -Información numérica indispensable (INI), en términos de las autoras- varios tipos de información adicional: 
- Eventos de iniciación (EI). Expresan sucesos que “inician” o "causan” el evento mencionado en la siguiente frase, generando así cadenas o vínculos causales entre los eventos descritos.

- Información del marco (IM). Aporta información acerca del personaje, el marco y el tema de la historia, y según las autoras, facilita que el niño ponga en marcha su conocimiento sobre el mundo real, y más concretamente, sobre el campo de conocimiento referido en el texto.

- Información de explicación (EX). Son explicaciones que explicitan las relaciones o consecuencias de los eventos que aparecen de manera implícita en el texto

- Información temporal (TE). Se corresponde con expresiones temporales o circunstanciales.

- Información narrativa (NA). Es información superflua y descriptiva que no proporciona ninguna ayuda real a la comprensión del enunciado ni a la resolución del problema.

Hemos incluido en la descripción de los tipos de información adicional unas iniciales entre paréntesis con el objetivo de que el lector pueda comprobar a qué tipo de información corresponde cada fragmento del ejemplo del problema reescrito resultante, que tomamos de las autoras, y que presentamos a continuación:

Un pastor está en una montaña (IM). El pastor quiere alimentar a sus ovejas (IM). El pastor tiene un rebaño de 30 ovejas (INI). En su rebaño el pastor tiene una oveja negra y una oveja blanca de Escocia (NA). Cada año (TE), se organiza un gran mercado en el pueblo (IE). El pastor compra algunas ovejas en el mercado (INI). Esto incrementa el tamaño de su rebaño (EX). Por la mañana (TE) el pastor se percata de la presencia de un oso en la montaña (IE). La noche siguiente (TE), el oso devora 12 ovejas (INI). Esto reduce el tamaño del rebaño (EX). Por la mañana (TE) el pastor cuenta sus ovejas (EX). Ahora hay 35 ovejas en el rebaño (INI). ¿Cuántas ovejas compró el pastor en el mercado? Moreau y Coquin-Viennot (2003), p. 114

Todos estos tipos de información adicional se desarrollaron considerando el modelo de Reusser (1988) para facilitar el análisis de la situación del problema y la creación del M.E.S.

A partir de estos problemas, las autoras comprobaron si los niños de $5^{\circ}$ curso generaban dos niveles complementarios de representación: modelo matemático del problema y modelo episódico de la situación. Para ello sometieron a los alumnos a dos tareas: la primera consistía en seleccionar la información para hacer el problema lo más corto posible, sin que éste perdiera su significado; en la segunda se les pedía que seleccionaran la información para hacer el problema más fácil de entender. Con la primera de las tareas se buscaban evidencias de la creación de un modelo matemático del problema, para lo cual los niños sólo necesitarían seleccionar la información numérica indispensable, prescindiendo del resto de la información, que aportaba un contexto situacional, pero que no aportaba ningún dato numérico al problema. Al plantear la segunda tarea se pretendía constatar la creación de un modelo de la situación, para lo cual los niños deberían elegir la información acerca de las cantidades de los conjuntos y las relaciones entre ellos, y además toda la información contextual excepto la información narrativa la cual, al ser información no relevante, no sería necesaria para la formación del modelo de la situación del problema.

Los resultados obtenidos apuntaban a que los alumnos, especialmente los más competentes, seleccionaban sólo información numérica imprescindible en la primera tarea, dirigida a la construcción del modelo matemático del problema, e información situacional, pero no narrativa, en la segunda, en la que se buscaba la generación del modelo episódico de la situación. A partir de estos resultados las autoras concluyeron que: 
(...) el hecho de especificar las relaciones entre los eventos o acciones contribuye a la comprensión de los problemas escritos. (...) La construcción de un modelo de la situación coherente constituye así un sustento adecuado para la comprensión de las relaciones entre los elementos que son indispensables para la resolución del problema. Moreau y Coquin-Viennot (2003), p. 119

Sin embargo, el estudio de estas autoras cuenta una limitación fundamental: la tarea no era la de resolver los problemas, de manera que no es posible saber, a partir de los resultados del estudio, si la generación de este M.E.S. revertía en una mejor comprensión del enunciado y si esto, a su vez permitía resolver los problemas con más éxito, en relación a su rendimiento en problemas "estándar".

Los estudios desarrollados por Vicente et al. (Vicente, 2006; Vicente et al., en prensa) tienen en cuenta estas limitaciones y, partiendo de problemas similares a los utilizados en el trabajo de Moreau y Coquin-Viennot (2003), diseñaron cuatro estudios en el que se comparaba el rendimiento de alumnos de $3^{\circ}$ a $5^{\circ}$ de Primaria en problemas de cambio "estándar" de dos operaciones, y de dos niveles de dificultad, con versiones reescritas matemática y situacionalmente. La reescritura matemática, idéntica en los cuatro estudios, resaltaba la estructura matemática del problema, tal y como se muestra en el ejemplo:

Pedro tenía 37 metros de cable. Compró X metros de cable más y los juntó con los que tenía. Del total de metros cable que juntó ha utilizado Y metros y le han sobrado 11 metros de cable. ¿Cuántos metros de cable compró/ha utilizado? Vicente, 2006, p. 185

Por otra parte, la reescritura situacional fue modificada a lo largo de los estudios. En el primero de ellos (Vicente, 2006; Vicente et al, en prensa) esta versión describía los personajes de la situación problemática, sus intenciones, y establecía una estructura causal y temporal de la situación problemática, siguiendo el modelo de Reusser (1988), y tal y como se muestra en el ejemplo (la información añadida se muestra en cursiva):

Pedro quería renovar la instalación eléctrica de su casa. Pedro tenía 47 metros de cable que le babian sobrado de una instalación anterior. Como Pedro se dio cuenta de que esos metros de cable no serían suficientes para toda la instalación compró X metros de cable más. Después de comprar el cable comenzó la instalación. Al hacer la instalación ha utilizado Y metros de cable, y cuando acaba comprueba que le han sobrado 11 metros de cable. Entonces Pedro se pregunta ¿Cuántos metros de cable compré/he utilizado? Vicente, 2006, p. 183

Los resultados del primer estudio mostraron que sólo la información matemática mejoró el rendimiento de los alumnos en los problemas difíciles, especialmente de los alumnos más pequeños. Las versiones situacionales de los problemas, en cambio, no mejoraron el rendimiento en los problemas difíciles, mientras que en los problemas fáciles fueron los que peor resultado suscitaron.

En vista de estos resultados, los autores establecieron cuatro explicaciones diferentes: primero, que la versión situacional resultante era demasiado extensa y compleja desde el punto de vista textual; segundo, que los problemas eran lo suficientemente sencillos desde el punto de vista situacional como para que la información situacional no fuera necesaria; tercero, que el formato -textual- de la tarea no favorecía la creación del modelo de la situación; y finalmente, que la resolución de problemas aritméticos no requiere de la creación de un modelo de la situación.

Para comprobar la primera explicación los autores diseñaron un segundo estudio (Vicente et al. en prensa) en el que se elaboraron tres versiones reescritas situacionalmente: una primera versión solamente con información temporal, una segunda solamente con información causal y una tercera con una combinación de ambas, tal y como muestran los ejemplos: 
Temporal: "Pedro tenía 47 metros de cable. Entonces compró X metros de cable más. Después de comprar el cable comenzó una instalación. Al hacer la instalación ha utilizado $\mathrm{Y}$ metros de cable, y cuando acaba le han sobrado 11 metros de cable.¿Cuántos metros de cable compró/ha utilizado"

Causal: "Pedro tenía 47 metros de cable que le habían sobrado de una instalación anterior. Como Pedro se dio cuenta de que esos metros de cable no serían suficientes para hacer una nueva instalación compró $\mathrm{X}$ metros de cable más. Como ba hecho una nueva instalación, ha utilizado Y metros de cable, y le han sobrado 11 metros de cable.¿Cuántos metros de cable compró/ha utilizado" Vicente, 2006, pp. 205-206

De esta manera, los autores pretendían preservar únicamente lo que Reusser (1988) consideraba la esencia del M.E.S.: la estructura temporal y causal de la situación, a la vez que se reducía considerablemente la extensión y complejidad textual del enunciado de los problemas. Los resultados mostraron que sólo las versiones temporales de los problemas fáciles suscitaron un ligero incremento del rendimiento de los alumnos, si bien este incremento estuvo lejos de la significatividad estadística.

Para comprobar la segunda explicación, esto es, si la falta de dificultad situacional era la causa de los resultados del primer estudio, los autores realizaron un tercer trabajo en el cual introdujeron una nueva variable respecto a los estudios anteriores: el nivel de dificultad situacional, operacionalizada a través de la modificación del orden en el que se enunciaban los eventos de la situación, tal y como hicieran Staub y Reusser (1992). De este modo, en este tercer estudio los problemas, además de fáciles y difíciles desde el punto de vista matemático, podrían ser fáciles o difíciles desde el punto de vista situacional. Los problemas situacionalmente fáciles describían los eventos del problema siguiendo el orden natural de los acontecimientos, mientras que los problemas situacionalmente difíciles presentaban esta secuencia de manera desordenada, tal y como se muestran en los ejemplos de las versiones situacionales correspondientes:

Situacionalmente fácil: Hace dos días Pedro tenía 47 metros de cable. Ayer Pedro compró X metros de cable más. Esta mañana comenzó una nueva instalación. Mientras hacía la instalación ha utilizado Y metros de cable, y cuando acabó le habían sobrado 11 metros de cable. ¿Cuántos metros de cable compró/ha utilizado?

Situacionalmente difícil: Ayer Pedro compró X metros de cable más de los que ya tenía. Esta mañana comenzó una nueva instalación. Mientras hacia la instalación ha utilizado Y metros de cable, y cuando acabó le habían sobrado 11 metros de cable. Si hace dos días Pedro tenía 47 metros de cable. ¿Cuántos metros de cable compró/ha utilizado? Vicente, 2006, pp. 225-226

En este estudio la reescritura situacional se limitó a la versión temporal de los problemas, dado el modo de operacionalizar la dificultad situacional y teniendo en cuenta los resultados de los estudios previos. Los resultados obtenidos en este tercer estudio replicaron los de los estudios anteriores: mientras que la información matemática mejoraba el rendimiento en los problemas matemáticamente difíciles (y también en los matemáticamente fáciles pero situacionalmente difíciles), la información temporal no mejoró el rendimiento de los alumnos en ningún caso.

Finalmente, los autores proponían como posible explicación a los resultados de sus estudios previos que el formato exclusivamente textual de la tarea experimental no era el mejor modo de promover la creación del modelo de la situación del problema. Para comprobar esta explicación realizaron un cuarto estudio en el, basándose en la idea propuesta por Schnotz (Schnotz, 2002, 2003; Schnotz y Barnett, 1999) de que los modelos mentales se construyen más fácilmente a partir de imágenes que de textos, propusieron dos formatos alternativos para la tarea: un primer formato exclusivamente textual, y un segundo formato en el que, además del texto escrito, se incluían dibujos. Las 
versiones reescritas fueron, de nuevo, matemáticas y situacionales (temporales), de manera que el número total de problemas fue de 2 (formato: con dibujo/ sin dibujo) X 2 (Dificultad: Fácil/ Difícil) X 3 (reescritura: estandar, matemática y situacional-temporal $)=12$ problemas. Los resultados obtenidos indicaron que los dibujos no ayudaban a la resolución de problemas, probablemente porque su diseño no fue el más apropiado. Sin embargo, el resultado más interesante de este estudio no estaba relacionado con el formato de la tarea, sino con la influencia de la información situacional. Este resultado fue que las versiones situacionales-temporales de los problemas suscitaron un nivel de acierto significativamente mayor que las versiones estándar. Los autores interpretaron estos resultados sosteniendo que en este estudio, a diferencia de los tres anteriores, los alumnos contaron con 4 versiones de los problemas con información matemática y se produjo un efecto de aprendizaje de los problemas con información matemática a los problemas situacionales-temporales, pero no a las versiones "estándar".

A la vista de los resultados de los cuatro estudios los autores plantearon como conclusión tentativa que la información situacional es únicamente útil cuando los alumnos disponen de claves matemáticas para interpretarlas. Dicho de otro modo, la creación de un modelo de la situación es un paso clave en la resolución de problemas siempre y cuando el alumno disponga del conocimiento matemático necesario para interpretar ese modelo desde el punto de vista matemático.

En resumen, de los principales estudios empíricos basados en la reescritura de problemas podemos destacar dos cosas. Por un lado, cada uno de los estudios que hemos descrito han elaborado una forma diferente de reescribir los problemas: sustituir el término "más que” por la expresión "cuántos.... no tendrán” (Hudson, 1984); incluir contextos situacionales ricos (Cummins et al., 1988); personalizar el problema con información significativa para los alumnos (Davis-Dorsey et al., 1991), incluir descripciones de situaciones competitivas entre personajes precediendo a problemas de comparación (Stern y Lehndorfer, 1992), indicar el estado inicial mediante una acción, alterar la estructura temporal de los hechos, otorgar siempre al protagonista del problema el rol sintáctico de sujeto en las oraciones del problema y evitar el uso de pronombres en problemas de cambio (Staub y Reusser, 1992), y finalmente, incluir información situacional de diferentes tipos en problemas de cambio de dos operaciones (Moreau y Coquin-Viennot, 2003; Vicente, 2006; Vicente et al., en prensa). Como podemos observar, la variabilidad de los criterios para reescribir los problemas es muy alta. Por otro lado, el segundo aspecto que queremos destacar, es el relativo a los resultados obtenidos: en tres de ellos (Davis-Dorsey et al., 1991; Hudson, 1983 y Stern y Lehndorfer, 1992) los resultados mostraron que los problemas reescritos eran más fáciles de resolver que los problemas “estándar". En el estudio de Cummins et al. (1988) y en tres de los cuatro estudios realizados por Vicente (2006), por el contrario, no encontraron diferencias entre los problemas reescritos y los problemas estándar, mientras que el estudio de Staub y Reusser (1992) sólo ofrece resultados especulativos acerca de la influencia relativa de algunas de las modificaciones que introdujeron y el de Moreau y Coquin-Viennot (2003) no permite extraer datos acerca de la influencia de la reescritura en la resolución de problemas ya que, si bien en este estudio la operacionalización de la reescritura situacional fue muy clara, mostraba una limitación fundamental: la tarea no era la de resolver el problema, de manera que no podemos saber si la construcción de un modelo de la situación dife- 
renciado del modelo matemático del problema tiene alguna influencia en el proceso de resolución.

Finalmente, los estudios realizados por Vicente et al. (Vicente, 2006; Vicente et al., en prensa) también definieron con nitidez la reescritura situacional, diferenciándola claramente de la reescritura matemática, y sometieron a los alumnos a la tarea de resolver los problemas, pero no se hallaron evidencias de la necesidad de generar un modelo de la situación. De hecho, los únicos resultados positivos encontrados a este respecto, en su último estudio, fueron resultados inesperados, debidos a un efecto de aprendizaje, de manera que el hallazgo de que sólo cuando los niños cuentan con claves matemáticas para interpretar la información temporal son capaces de utilizarla para resolver el problema con más eficiencia requiere estudios adicionales que lo corroboren.

\section{Conclusiones e implicaciones educativas}

La tarea de resolver un problema de matemáticas implica la interacción de varios tipos de procesos cognitivos. Además del componente matemático, esto es, la comprensión de la estructura matemática del problema, algunos modelos teóricos (Kintsch, 1988; Nathan et al., 1992; Reusser, 1988; Verschaffel et al., 1994) proponen que, para resolver correctamente los problemas, es necesario generar una representación mental cualitativa de la situación descrita por el problema, utilizando para ello el conocimiento sobre el mundo real de que disponga el sujeto. Esta afirmación, que ha demostrado ser cierta para los denominados "problemas realistas" y para los problemas de álgebra, no ha sido corroborada claramente para los problemas aritméticos, tal y como se desprende del análisis de los estudios empíricos basados en la reescritura de problemas que hemos recogido en este trabajo. La mayor parte de esos estudios, al reescribir situacionalmente los problemas, modificaron determinados aspectos matemáticos de los mismos, de manera que no puede concluirse que esta mejora se deba a una mejor comprensión de la situación descrita por el problema. Además, aquellos estudios que han diferenciado claramente ambos tipos de reescritura (matemática y situacional) no han apoyado la idea de que crear un modelo del problema sea necesario para resolverlo.

¿Significa esto que la información situacional es irrelevante para la tarea, y que crear un modelo de la situación no es necesario para la resolución de problemas aritméticos? Nuestra respuesta es que no. La limitación que acabamos de señalar, esto es, la mezcla de los dos tipos de rescritura en la mayor parte de los estudios, unido al hecho de que en la mayoría de ellos se hayan encontrado resultados positivos, apunta a la idea de que si bien la información situacional por sí misma no es útil para que los alumnos resuelvan con mayor acierto los problemas, cuando esta información situacional permite hacer inferencias sobre la estructura matemática del problema, como en el caso de Hudson (1983), Stern y Lehrndorfer (1992) y Vicente (2006), permite a los alumnos mejorar su rendimiento.

De esta manera, las conclusiones que podemos extraer de esta revisión son dos. La primera conclusión de ellas es que si bien la creación de un modelo de la situación se ha mostrado necesaria para la resolución de problemas realistas y algebraicos, para los problemas aritméticos la creación de este modelo de la situación probablemente contribuya a la mejor resolución del problema sólo cuando contenga claves situacionales de la estructura matemática del problema, como información sobre la magnitud relativa de los conjuntos 
en los problemas de comparación (Stern y Lehrndorfer, 1992) o como la evolución temporal de las magnitudes en los problemas de cambio (Vicente, 2006).

De esta primera conclusión podemos extraer una segunda: el modelo de la situación que permite elaborar las inferencias necesarias para generar el modelo matemático del problema difiere en función del tipo de problema a resolver. Esto es, mientras que la inclusión de vínculos causales entre la situación comparativa y las cantidades del problema ha mostrado ser mas eficaz para los problemas de comparación, tal y como se desprende de los resultados del estudio de Stern y Lehrndorfer ("Pedro es el hermano mayor de Laura. Como es mayor, su habitación es mayor..."), la explicitación de la estructura temporal parece más adecuada para los problemas de cambio, como se desprende del trabajo de Vicente (2006).

¿Qué implicaciones pueden tener estas conclusiones sobre la labor educativa de los maestros? Para responder a esta pregunta debemos considerar dos elementos: el tipo de tareas a las que se enfrentan los alumnos y el modo de abordarlas en el aula. En las primeras páginas de este trabajo proponíamos la idea de que los problemas de matemáticas con los que se encuentra un alumno a lo largo de su escolaridad pueden situarse en un continuo, desde la resolución de operaciones, en las que únicamente se necesitan conocimientos matemáticos, hasta los problemas realistas, en los que la aplicación de estos conocimientos matemáticos es claramente insuficiente para la resolución con éxito del problema. Entre esos dos polos se situarían los problemas de álgebra y los aritméticos.

Por otra parte, la falta de éxito de los alumnos en los problemas realistas parece estar causada, al menos en parte, por la cultura del aula, según la cual todo problema de matemáticas tiene una solución, que es única y de carácter exclusivamente matemático (Verschaffel, 2002). De esta manera, la forma de resolver los problemas en el aula se sustenta en la idea implícita de que resolver problemas es, en última instancia, resolver la operación aritmética subyacente al mismo, y que para ello la única información necesaria es la información matemática. Así, el conocimiento del mundo real y las consideraciones que, desde el sentido común, pudiera realizar el alumno, quedan fuera del proceso de resolución, de tal modo que, al final, las matemáticas terminan siendo ajenas al mundo real. Este hecho, ampliamente documentado para los problemas realistas, probablemente sea la causa del bajo rendimiento de los alumnos en problemas similares a los utilizados por el informe PISA (2003) en los que, tal y como señala el Instituto Nacional de Evaluación y Calidad del Sistema Educativo (INECSE), "no se evaluaba simplemente la capacidad de los alumnos para realizar operaciones matemáticas, sino el grado en que son capaces de reconocer, formular y abordar problemas matemáticos en contextos reales" (INECSE, 2004, p. 2).

¿Cuál es el origen de esta visión distorsionada de los problemas de matemáticas? Retornando la idea del continuo de las tareas matemáticas a las que se enfrenta el alumno a lo largo de su escolaridad, los primeros problemas de matemáticas a los que se enfrenta son los problemas aritméticos. Con frecuencia, estos problemas aritméticos son resueltos sin apelar a otro conocimiento que no sea el matemático, de una manera muy similar a la resolución de operaciones aritméticas, fundamentalmente por dos razones. La primera probablemente sea la que hemos visto en la revisión de los estudios empíricos: no está claro que comprender la situación empleando para ello conocimientos sobre el mundo real ayude a resolver mejor los problemas, mientras que las ayudas matemáticas sí han probado ser eficaces. De esta manera, es 
muy lógico que los maestros se apoyen en lo que ayuda a sus alumnos a resolver mejor los problemas (ayudas matemáticas) y releguen a un segundo plano lo que no es tan útil (ayudas situacionales).

La segunda causa podemos encontrarla en el modo en que los libros de texto presentan la resolución de problemas. Tal y como señalan Orrantia, González y Vicente (2005), los libros de texto suelen presentar la resolución de problemas como meros ejercicios de ejecución de operaciones. Además, los problemas suelen pertenecer principalmente a los tipos de problemas más sencillos (Riley et al., 1983), y rara vez incluyen información situacional, de manera que toda la información que aparece en el problema es información matemática útil para resolverlo, y toda la información necesaria para resolverlo está presente en el problema. De esta manera, los problemas suelen ser meros ejercicios estereotipados para la aplicación de operaciones aritméticas.

De esta manera, el primer contacto con la resolución de problemas, a través de los problemas aritméticos, hace que se establezca un modo de resolver problemas que, por un lado, se apoya fundamentalmente en el uso exclusivo de conocimientos matemáticos, y que por otro tiende a afrontar los problemas de manera estereotipada. Una vez que el alumno ha aprendido a resolver los problemas de ese modo, con toda seguridad generalizará ese aprendizaje a la resolución del resto de problemas, esto es, a los problemas algebraicos y realistas, dando como resultado un divorcio creciente entre las matemáticas de la escuela, en las que con lo matemático es suficiente, y la vida real, llena de matices que requieren la aplicación de otros tipos de conocimientos.

Teniendo en cuenta lo anterior, es evidente que para mitigar los efectos de esta disociación entre el mundo real y las matemáticas, en primer lugar debería supeditarse la ejecución de operaciones a la resolución de problemas, y no a la inversa. En segundo lugar, la resolución de problemas debería dar cabida a la aplicación de lo que el alumno ya sabe sobre el mundo real. Como hemos visto, esto es más sencillo para los problemas realistas y de álgebra, en los que los errores cometidos por la inhibición de consideraciones realistas son más evidentes, pero ¿qué hacer con los problemas aritméticos, en los que la inclusión de información que active los conocimientos previos sobre el mundo real no ha demostrado ser tan eficaz como las ayudas matemáticas? Una respuesta razonable sería alejarnos de estos problemas estereotipados y al servicio de la ejercitación de las operaciones aritméticas y proponer problemas de todos los tipos (cambio, comparación y combinación), y que contengan información relativa a la situación del problema que permita al alumno resolverlo con más éxito. Como hemos señalado en la segunda de las conclusiones de nuestra revisión, sobre cada tipo de problema probablemente pueda generarse un modelo de la situación cualitativo, con claves contextuales ligadas a la estructura matemática del problema (cadenas causales para los problemas de comparación y temporales en los de cambio, por ejemplo), que puedan ser interpretadas por los alumnos para resolver el problema. Ayudarles a detectar y a interpretar estas claves contextuales en los problemas aritméticos, que son los primeros a los que se enfrentan, puede ser el primer paso para que, en problemas contextualmente más complejos, como los problemas de álgebra o realistas, no se limiten a ejecutar operaciones y a proponer soluciones matemáticas que, en muchos casos, carecen de sentido. Esto es, enseñando a los niños a apoyarse en claves contextuales útiles para resolver los problemas aritméticos estarán aprendiendo que la clave de los problemas de matemáticas no tiene por qué estar únicamente en la aplicación de conocimientos matemáticos, rompiendo así la concepción errónea 
que está en la base de la mayor parte de los errores cometidos por los alumnos en los problemas de álgebra y realistas.

Una primera aproximación eficaz a este modo de resolver los problemas podría ser la elaboración de versiones reescritas en las que se incluyan claves situacionales vinculadas al modelo del problema. Por ejemplo, podrían incluirse situaciones comparativas con vínculos causales relacionados con el modelo del problema, de manera similar al estudio de Stern y Lehndorfer (1992) para problemas de comparación, y enseñar al alumno a utilizar estas claves como pistas para delimitar el modelo del problema y, por lo tanto, para decidir qué operación hay que realizar para resolverlo. Evidentemente, la reescritura es una ayuda textual cuya eficacia dependerá en buena parte de la relevancia que el maestro le dé en el proceso de resolución de problemas. Nuestra propuesta es incluir este razonamiento no estrictamente cuantitativo dentro del proceso de resolución de problemas aritméticos para, después, generalizarlo a la resolución de otros tipos de problemas, como los de álgebra y los realistas.

\section{Notas}

* Santiago Vicente y José Orrantia son miembros del Departamento de Psicología Evolutiva y de la Educación de la Universidad de Salamanca. Sus campos de investigación son: a) el estudio de los procesos cognitivos implicados en la resolución de problemas y en el cálculo; b) el desarrollo del conocimiento matemático; c) el análisis de la interacción en sesiones de resolución de problemas de matemáticas; y d) las dificultades de aprendizaje del cálculo y la resolución de problemas y su intervención

El modelo de Kintsch y Greeno (1985) también generaba una serie de inferencias, a partir de los conocimientos previos del modelo, necesarias para mantener la coherencia del texto. Sin embargo, el modelo de Kintsch, además de estas inferencias, generaba una cantidad de proposiciones mucho mayor que el modelo anterior

Lamentablemente, esa es la única información que aportan del resultado del análisis proposicional de los enunciados de los problemas, ya que no mencionan nada de la estructura del texto ni de como esas proposiciones se relacionaban entre sí.

\section{Referencias}

BAROODY, A. J. (1993). The relationship between the order-irrelevance principle and counting skill. Journal for Research in Mathematics Education, 24 (5), 415-427.

BAROODY, A. (1999). Children's relational knowledge of addition and subtraction. Cognition and Instruction, 17 (2), $137-175$.

BAROODY. A. J. \& GANNON,K. E. (1984). The development of commutativity principle and economical addition strategies. Cognition and Instruction, 1, 321-329.

BaroODY, A. J., WILKINS, J. L. M. \& Tillikainen, S. H. (2003). The development of children's understanding of additive commutativity: From protoquantitative concept to general concept. En A. J. Baroody \& A. Dowker (Eds.), The development of arithmetic concepts and skills: Constructing adaptive expertise (pp. 127-160). Mahwah, NJ: Erlbaum.

BRIARS, D. J. \& LARKIN, J. H. (1984). An integrated model of skill in solving elementary word problems. Cognition and Instruction, 1, 245-296.

CALDWELL, L. (1995). Contextual considerations in the solution of children's multiplication and division word problems. Tesis de graduación no publicada. Queens University, Belfast, Irlanda del Norte

Canobi, K., ReEve, R. \& Pattison, P. (2002). Young children's understanding of addition concepts. Educational Psychology, $22(5), 513-532$.

Carpenter, T. P., Lindquist, M. M., Matthews, W. \& Silver, E. A. (1983). Results of the Third NAEP Mathematics Assessment: Secondary School. Mathematics Teacher, 76, 652 - 659.

Cummins, D. D., Kintsch, W., Reusser, K. \& Weimer, R. (1988). The role of understanding in solving word problems. Cognitive Psychology, 20, 405-438.

DAVIS-DORSEY, J., Ross, S. M. \& Morrison, G, R. (1991). The role of rewording and context personalization in the solving of mathematical word-problems. Journal of Educational Psychology, 83, 61-68.

De CoRte, E., Verschaffel, L. \& DE WIN, L. (1985). Influence of rewording verbal problems on childrens problem representations and solutions. Journal of Educational Psychology, 77, 460-470.

FAN, N., Mueller, J. H. \& MARINI, A. (1994). Solving difference problems: wording primes coordination. Cognition and Instruction, $12(4), 355-369$.

FusON, K. (1988). Children's counting and concepts of number. Nueva York: Springer-Verlag.

FUSON, K. C. (1992). Research on whole number addition and subtraction. En D. Grouws (Ed.), Handbook of research on mathematics teaching and learning (pp. 243-275). Nueva York: Macmillan.

FusON, K., SECADA, W. \& HALL, J. (1983). Matching, counting, and conservation of numeral equivalence. Child Development, 54, $91-97$.

Fuson, K., Wearne, D., Hiebert, J., Murray, H., Human, P., Olivier, A., Carpenter, T. P. \& Fennema, E. (1997). Children's conceptual structures for multidigit numbers and methods of multidigit addition and subtraction. Journal for Research in Mathematics Education, 28 (2), 130-162. 
GreER, B. (1993). The modeling perspective on wor(l)d problems. Journal of Mathematical Behavior, 12, 239-250.

Heller, J. I. \& GREeNO, J. G. (1978). Semantic processing in arithmetic word problem solving. Paper presentado en Midwestern Psychological Association Convention. Chicago.

HidALGo, M. C. (1997). L'activation des connaissances à propos du monde réel dans la résolution de problèmes verbaux en arithmétique. Tesis Doctoral no publicada Université Laval, Québec, Canada.

Hudson, T. (1983). Correspondences and numerical differences between disjoint sets. Child Development, 54, 84-90.

Instituto NaCional De Evaluación y CALIDAD DEL Sistema EduCativo (INECSE) (2004). Aprender para el mundo de mañana. Resumen de resultados. PISA 2003. Madrid: Ministerio de Educación y Ciencia

JoNASSEN, D. H. (2003). Designing research-based instruction for story problems. Educational Psychology Review, 15 (3), $267-$ 295.

KINTSCH, W. (1988). The role of knowledge in discourse comprehension: a Construction-Integration model. Psychological Review, 95 (2), 163-182.

KINTSCH, W. (1998). Comprehension: a paradigm for cognition. Cambridge: Cambridge University Press.

KINTSCH, W. \& GREENO, J. (1985). Undertanding and solving word arithmetic problem. Psychological Rewiev, 92, 109-129.

MAYER, R.E. (1981). Frecuency norms and structural analysis of algebra story problems into families, categories and templates. International Science, 10, 135-175

Mayer, R. E., Fennell, S., Farmer, L. \& CAmpbell, J. (2004). A personalization effect in multimedia learning: Students learn better when words are in conversational style rather than formal style. Journal of Educational Psychology, 96 (2), 389395.

MOREAU, S. \& COQuin-VIENNOT, D. (2003). Comprehension of arithmetic word problems by fifth-grade pupils: representations and selection of information. British Journal of Educational Psychology, 73, 109-121

Nathan, M. J., KinTSCH, W. \& Young, E. (1992). A theory of algebra-word problem comprehension and its implications for the design of Learning Environments. Cognition and Instruction, 9 (4), 329-389.

OrRANTIA, J. (2003). El rol del conocimiento conceptual en la resolución de problemas aritméticos con estructura aditiva. Infancia y Aprendizaje, 26 (4), 451-468.

OrRANTIA, J., GONZÁLEZ, L. B. \& VICENTE, S. (2005) Un análisis de los problemas aritméticos en los libros de Educación Primaria. Infancia y Aprendizaje, 26 (4), 429-451.

RadATz, H. (1983). Untersuchungen zum Lösen eingekleideter Aufgaben. Zeitschrift für Mathematik-Didaktik, 4, $205-217$

ReNKL, A. (1999). The gap between school and everyday knowledge in mathematics. Paper ppresentado en la Eighth European Conference for Research on Learning and Instruction, Göteborg, Suecia, Agosto.

ResNiCK, L. (1983). A developmental theory of number understanding. En I. Gingsburg \& T. Herbert, The development of mathematical thinking (pp. 109-151). Londres: Academic Press.

ReSNICK, L. (1989). Developing mathematical knowledge. American Psychologist, 44 (2), 162-169.

RESNICK, L. (1992). From protoquantities to operators: Building mathematical competence on a foundation of everyday knowledge. En G. Leinhardt, R. Putnam \& R. A. Hattrup (Eds.), Analysis of arithmetic for mathematics teaching (pp. 373429). Hillsdale, NJ: LEA.

Reusser, K. (1988), Problem Solving Beyond the Logic of Things: Contextual Effects on Understanding and Solving Word Problems, Instructional Science, 17, 309-338.

ReusSER, K. Y STEBLER, R. (1997). Every word problem has a solution. The social rationality of mathematical modeling in schools. Learning and Instruction, 7, 309-327.

RiLEY, N. S., GREENO, J. \& HeLLER, J. I. (1983). Development of children's problem solving ability in arithmetic. En H. P. Ginsburg (Ed.), The development of mathematical thinking (pp. 153-196). Nueva York: Academic Press.

SÄLJÖ, R. \& WYNDHAMn, J. (1990) Problem solving, academic performance, and situated reasoning: A study of joint cognitive activity in the formal setting. British Journal of Educational Psychology, 60 (3), $245-54$.

SCHNOTZ, W. (2002). Towards an Integrated View of Learning from Text and Visual Displays. Educational Psychology Review, $14(2), 101-120$.

SCHNOTZ, W. (2003). External and Internal Representations in Multimedia Learning. Learning and Instruction, 13 (2), $117-$ 23.

SCHNOTZ, W. \& BARNETT, M. (1999). Construction and interference in learning from multiple representation. Learning and Instruction, 13, 141-156.

SEMADENI, Z. (1995). Developing children's understanding of verbal arithmetical problems. En M. Hejn? \& J. Novotná (Eds.), Proceedings of the International Symposium on Elementary Math Teaching (pp. 27-33). Praga: Faculty of Education, Charles University

Silver, E. A., Shapiro, L. J. \& DeUTSCH, A. (1993). Sense making and the solution of division problems involving remainders: An examination of middle school students' solution processes and their interpretations of solutions. Journal for Research in Mathematics Education, 24 (2), 117-135.

SOPHian, C., HaRley, H. \& MARTin, C. S. (1995). Relational and representational aspects of early number development. Cognition and Instruction, 13 (2), 253-268.

StAub, F. \& Reusser, K. (1992). The role of presentational factors in understanding and solving mathematical word problems. Paper presentado en el Meeting of the American Educational Research Association, San Francisco.

StAub, F. C. \& Reusser, K. (1995). The role of presentational structures in understanding and solving mathematical word problems. En C. A. Weaver III, S. Mannes \& C. R. Fletcher (Eds.), Discourse Comprehension: Essays in honor of Walter Kintsch (pp. 285-305). Hillsdale, NJ: Lawrence Erlbaum.

Stern, E. \& LeHrndorfer, A. (1992). The role of situational context in solving word-problems. Cognitive Development, 7 , 259-268.

VAN DIJK, T. A. \& KINTSCH, W. (1983). Strategies of discourse comprehension. Nueva York: Academic Press.

VERSCHAFFEL, L. (2002). Taking the modelling perspective seriously at the elementary school level: promises and pitfalls. En A Cockburn \& E. Nardi (Eds.), Proceedings of the $26^{\text {th }}$ Annual Conference of the International Group for the Psychology of Mathematics Education (Vol. 1, pp 64-82). Norwich: School of Education and Professional Development, University of East Anglia, U.K.

Verschaffel, L., De Corte, E. \& Lassure, S. (1994). Realistic considerations in matyhematical modelling of school word problems. En W. Schnotz, S. Vosniadou \& M. Carretero, (Eds), New perspectives on conpcetual change (pp. 175-189). Oxford: Elserver. 
Verschaffel, L., De CoRTe, E. \& Lasure, S. (1999). Children's conceptions about the role of real-world knowledge in mathematical modelling of school word problems. En W. Schnotz, S. Vosniadou \& M. Carretero (Eds.), New perspectives on conceptual change (pp 175-189). Oxford: Elsevier.

Verschaffel, L., Greer, B. \& De Corte, E. (2000). Making sense of word problems. Lisse: Swets \& Zeitlinger Publishers.

VICENTE, S. (2006). Conocimiento matemático y situacional y su influencia en la resolución de situaciones problemáticas de estructura aditiva. Tesis doctoral no publicada. Universidad de Salamanca

Vicente, S., OrRantia, J. \& VerschafFel, L. (en prensa). Influence of situational and conceptual rewording on word problem solving. British Journal of Educational Psychology.

Yoshida, H., Verschaffel, L. \& De CORTE, E. (1997). Realistic considerations in solving problematic word problems: Do Japanese and Belgian children have the same difficulties? Learning and Instruction, 7, 329-338. 